\section{Quantum Critical Point Found for Iron Arsenide Superconductors}

New experiments on a recently discovered class of iron-based superconductors suggest that the ability of their electrons to conduct electricity without resistance is directly connected with the magnetic properties of those electrons.

Results of the experiments appear in the January 8 issue of Physical Review Letters (DOI: 10.1103/PhysRevLett.104. 017204). The tests, which were carried out by a team of U.S. and Chinese researchers, shed light on the fundamental nature of high-temperature superconductivity, said Rice University researcher Qimiao $\mathrm{Si}$, a co-author on the study.

If better understood, high-temperature superconductors could be used to revolutionize electric generators, MRI scanners, high-speed trains, and other devices.

In the study, researchers from Rice University, the University of Tennessee, Oak Ridge National Laboratory (ORNL), the National Institute of Standards and Technology (NIST), the Chinese Academy of Sciences' Institute of Physics, and Renmin University in Beijing examined several ironarsenide compounds. These are the undoped parents of the iron pnictides, a class of materials that were found to be hightemperature superconductors in 2008.

The experiments set out to test theoretical predictions that $\mathrm{Si}$ and collaborators published in the Proceedings of the National Academy of Sciences last year. They predicted that varying the size of some atoms in the parent compounds could allow researchers to tune the material's quantum fluctuations. These types of fluctuations can create tipping points called magnetic "quantum critical points," a state that exists when a material is at the cusp of transitioning from one quantum phase to another.

Using neutron-scattering facilities at NIST and ORNL, the team bombarded the materials with neutrons to decipher their structural and magnetic properties. The tests, which supported Si's theoretical predictions, determined that the strength of magnetic order in the materials was reduced when arsenic atoms were replaced with slightly smaller phosphorus atoms.

"We found the first direct evidence that a magnetic quantum critical point exists in these materials," Si said.

The results were made possible by the efforts of N. Wang, from the Chinese Academy of Sciences' Institute of Physics, and his research group. They created a series of $\mathrm{CeFeAs}_{1-x} \mathrm{P}_{x} \mathrm{O}$ samples with varying amounts of phosphorous substituting for arsenic.

The discovery of high-temperature superconductivity in copper-oxide ceramics in 1986 led researchers to realize that quantum effects in electronic materials were far more complex than anticipated. One of these effects is quantum criticality. Criticality occurs near a tipping point that a material goes through when it changes phases. Many phase changes-like ice melting into water-occur because of thermal fluctuations. But quantum criticalities and quantum phase changes arise solely from quantum fluctuations.

"Our finding of a quantum critical point in iron pnictides opens the door for new avenues of research into this important class of materials," said University of Tennessee/ORNL researcher Pengcheng Dai, a neutron scattering specialist.

Si said, "The evidence from this study bolsters the hypothesis that high-temperature superconductivity in the iron pnictides originates from electronic magnetism. This should be contrasted to conventional lowtemperature superconductivity, which is caused by ionic vibrations."

\section{Transparent, Unipolar, QD-Based LEDs Operate in Air}

Due to their high efficiencies and tunable band emission, quantum dots (QDs) have great potential for being the active layer in light-emitting devices for many applications including solid-state lighting and advanced display technologies. The current challenges involved with using quantum dots in lightemitting devices are achieving device stability and aligning energy bands between charge transport layers and QD emitters. V. Wood, V. Bulovic, M.G. Bawendi, and colleagues at the Massachusetts Institute of Technology have recently created allinorganic, unipolar, QD-based light-emitting devices (QD-LEDs). The device design simplifies energy band alignment by using only electrons as the charge carrier type while also being able to operate in air even un- packaged due to all-inorganic constituents.

In the January 13 issue of Nano Letters (DOI: 10.1021/n1902425g; p.24), the research group reports on these transparent QD-LED structures based on $n-i-n$ unipolar architecture, using only $n$-type charge transport layers, which differs from traditional diodes using the $p-n$ junction. The reported structure allows for the electroluminescence from colloidally synthesized QDs of multiple colors. Modifying the size and makeup of the QDs can tune their emission wavelength.

The QD-LEDs are made on transparent conducting indium-tin-oxide (ITO)-coated glass. The basic device structure consists of a charge transport layer of alloyed $\mathrm{ZnO}$ and $\mathrm{SnO}_{2}$ (ZTO), a QD emissive layer, another layer of ZTO, and a transparent top electrode made of ITO similar to the bottom electrode. The QDs are CdSe/ZnS or CdZeSe/CdZeS core/shell structures that emit red and green light, respectively. These QDs have high photoluminescent quantum yields of $90 \%$ and $70 \%$. Incorporating a layer of $\mathrm{ZnS}$ into the charge transport layer serving as an electronblocking layer limits the charging of the QD layer, providing stable luminance over time and enhancing overall device performance. The optimized device has optical absorption in the visible spectrum less than $4 \%$ on average, which is attributed to the QD layer, and optimized external quantum efficiency of $0.15 \%$. The lack of molecular organic charge transport layers and usage of only inorganic materials contribute to the device stability when operating in air without packaging.

CHARLES BROOKS

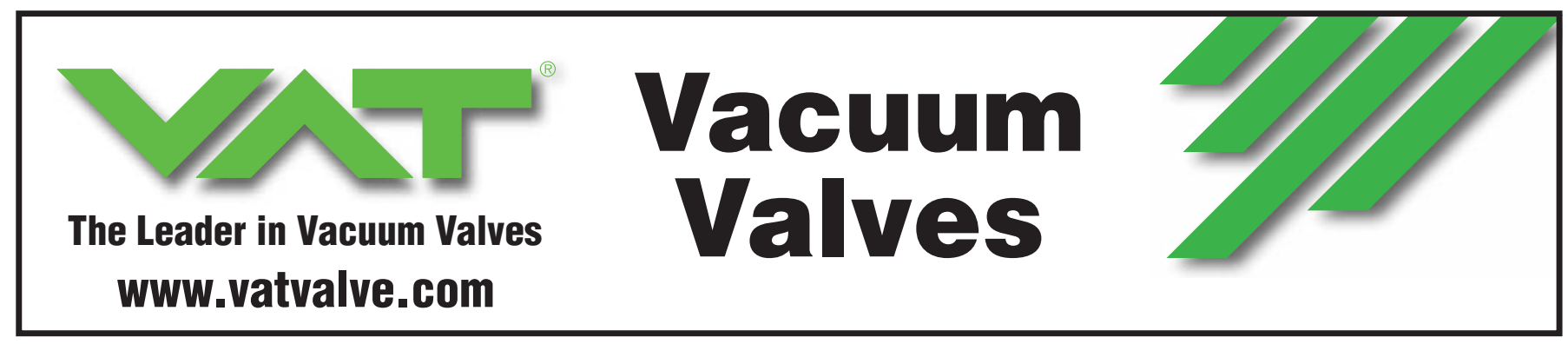

